Research Article

\title{
What is the impact of obesity on adolescent pregnancy?
}

\author{
Abstract \\ Study objective: To evaluate the impact of obesity on delivery route and neonatal outcomes \\ in adolescents at our tertiary care facility \\ Design: Retrospective cohort \\ Setting: Vidant Medical Center, a tertiary care facility serving twenty-nine counties in \\ eastern North Carolina
}

Participants: Adolescent pregnant patients, ages thirteen to nineteen, who delivered viable, singleton infants from January 1, 2009, to December 31, 2011

Interventions: Not applicable

Main outcome measures: Route of delivery, gestational age at time of delivery, neonatal weight, and Apgar scores

Results: One thousand thirty-four adolescents met the inclusion criteria and were separated into three categories based on their body mass index-for-age percentiles: normal weight $(\mathrm{N}$ $=220)$, overweight $(\mathrm{N}=328)$, and obese $(\mathrm{N}=486)$. Obese adolescents were at significantly increased risk for Cesarean delivery compared with their normal and overweight counterparts $(\mathrm{P}<.001)$. Normal weight adolescents were more likely to deliver preterm $(\mathrm{P}$ $<.001)$ and have low birth weight infants $(<2500 \mathrm{~g})(\mathrm{P}<.001)$, whereas obese adolescents were more likely to give birth to macrosomic infants $(\mathrm{P}<.001)$. Mean Apgar scores at five minutes did not differ significantly between the groups.

Conclusions: The weight of adolescents at time of delivery significantly impacts pregnancy outcomes. Obese adolescents in our study demonstrated some protection from risks of preterm delivery and low birth weight infants, however, they were at increased risk for macrosomia and Cesarean section.

Keywords: adolescent pregnancy, teen pregnancy, obesity
Volume 6 Issue $6-2017$

\author{
TL Hall,' JC Phipps, ${ }^{2}$ CA Hodson' \\ 'Department of Obstetrics \& Gynecology, Brody School of \\ Medicine at East Carolina University, USA \\ ${ }^{2}$ Obstetrics \& Gynecology Residency Program at Vidant Medical \\ Center, USA
}

Correspondence: Tana L Hall, MD, Brody School of Medicine at East Carolina University, Department of Obstetrics \& Gynecology, MA 176, 600 Moye Blvd, Greenville, NC, USA, Tel 252-744-5903, Fax 252-744-138I, Email hallt@ecu.edu

Received: April 27, 2017 | Published: June 22, 2017
Abbreviations: BMI, body mass index; CDC, center for disease control; NCSS, number cruncher statistical system

\section{Introduction}

Adolescent pregnancy continues to be a concern in our communities. Although teen birth rates have decreased by twenty-five percent from 2007 to 2011, the teen live birth rate in North Carolina was as high as 49.9 per 1000 teens in $2011 .{ }^{1}$ A number of these births are to adolescent mothers who already have one or more children. Research has shown that adolescent pregnancy impacts both the fetus and the mother. Infants born to adolescent mothers are at increased risk for prematurity, low birth weight, fetal death, neonatal death, and infant death. ${ }^{2,3}$ Pregnant adolescents are at increased risk for preeclampsia, retained postpartum weight, and poor social outcomes.,

A growing and complicating factor in adolescent pregnancy is obesity. Adolescent obesity has quadrupled in the past thirty years from five percent in 1980 to twenty-one percent in 2012, and now more than one third of adolescents are overweight or obese. ${ }^{6}$ We know that infants born to obese mothers are at increased risk for prematurity, macrosomia with possible birth injury, and fetal death. ${ }^{7}$. We also know that in adult patients, obesity increases the risk of gestational diabetes, pre-eclampsia, Cesarean delivery, and postpartum weight retention. ${ }^{8}$ This raises the question, does obesity in adolescent pregnancy convey the same risks demonstrated in adult pregnancy?
As we continue to look at adolescent pregnancy, we must begin to consider whether obesity further compounds the maternal and fetal risks we know to be associated with pregnancy in this population. The purpose of this study is to evaluate the impact of obesity on delivery route and neonatal outcomes in adolescents at a tertiary care facility.

\section{Materials and methods}

This is a retrospective cohort study of adolescent pregnant patients, ages thirteen to nineteen, who delivered viable, singleton infants at Vidant Medical Center from January 1, 2009, to December 31, 2011. Vidant Medical Center is our tertiary care facility that services twenty-nine counties in eastern North Carolina. Institutional review board approval was obtained, and the following data were collected from electronic medical records: age, race, weight and height at time of admission, route of delivery, gestational age at time of delivery, neonatal weight, and Apgar scores. Data were entered into a secure Excel database.

For adolescents through age twenty, the Center for Disease Control (CDC) recommends using body mass index-for-age percentiles rather than body mass index (BMI) alone, allowing interpretation of BMI relative to other adolescents of the same gender and age. ${ }^{7}$ In this study, BMI were calculated from recorded weights and heights at time of admission. Using the CDC published BMI-for-age percentiles chart for girls ages two to twenty years, study patients were divided into three categories: normal weight, overweight, and obese. 
Statistical methods utilized include Chi square analysis and one way analysis of variance using the Number Cruncher Statistical System (NCSS) 2007 statistical analysis system. Results were considered significant when $\mathrm{P}$ values were $<.05$.

\section{Results and discussion}

One thousand fifty adolescents, ages thirteen to nineteen, delivered viable, singleton infants during the three year timeframe of this study. Sixteen were excluded due to incomplete documentation in medical records. One thousand thirty-four patients remained in the study. As previously described, patients were separated into three categories based on their BMI-for-age percentile: normal weight $(\mathrm{N}=220)$, overweight $(\mathrm{N}=328)$, and obese $(\mathrm{N}=486)$. Demographics of the three groups were compared (Table 1). The mean age of the normal weight adolescents was 18.1 years versus mean ages of 17.8 years in both the obese and overweight groups, $\mathrm{P}<.05$ ). There were no other demographical differences between the groups.

Route of delivery was recorded as vaginal delivery, including operative vaginal delivery, or Cesarean delivery. Initial analysis of all adolescents in the study demonstrated that obese adolescents were at significantly increased risk for Cesarean delivery $(\mathrm{P}<.001)$ (Figure 1). The operative reports for all Cesarean deliveries were reviewed and categorized by indication: prior Cesarean delivery, arrest of labor, fetal malpresentation, fetal tracing, and other. Although they were not statistically significant, there were some noticeable differences in the indications (Figure 2). Obese adolescents had more repeat Cesarean deliveries than either the normal weight or overweight groups $(17.1 \%$ versus $6.43 \%$ in overweight and $5.7 \%$ in normal weight teens), and obese and overweight teens appeared more likely to arrest in labor (23.1\% and $21.3 \%$, respectively, versus $11.4 \%$ in normal weight teens). Fetal tracing concerns were the most common indication for Cesarean delivery in overweight adolescents compared with normal weight and obese adolescents $(36.2 \%$ versus $22.0 \%$ and $28.6 \%$, respectively).

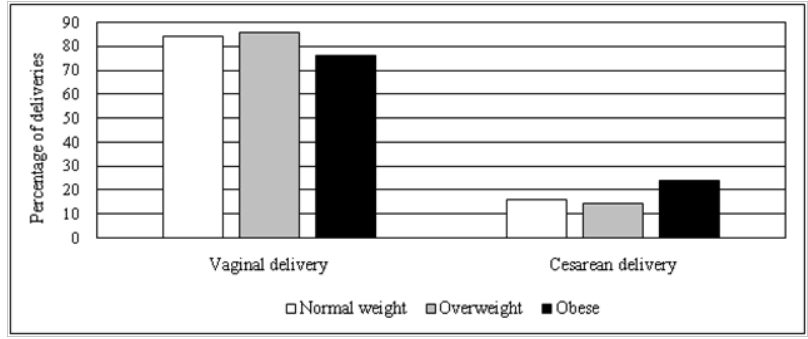

Figure I Route of delivery of all patients by weight classification. Chi-square 13.967, Degrees of freedom 2, Probability level $<.00 \mathrm{I}$.

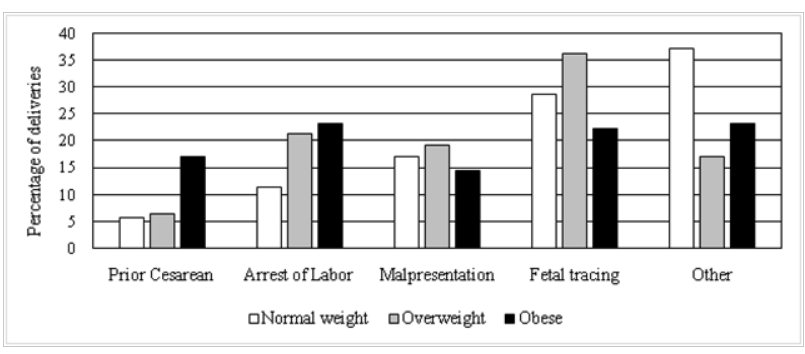

Figure 2 Indication for Cesarean delivery by weight classification. Chi-square 12.915, Degrees of freedom 8, Probability level .I I5.

Because a prior delivery, whether vaginal or Cesarean, may influence route of delivery for a subsequent pregnancy, a subset analysis was performed on the 884 primiparous patients in the study.
The primiparous patients were comprised of 180 normal weight adolescents, 286 overweight adolescents, and 418 obese adolescents. Obese adolescents remained at significantly increased risk for Cesarean delivery $(23.2 \%)$ compared with normal weight $(16.1 \%)$ and overweight teens $(15.4 \% ; \mathrm{P}=.02)$ based on analysis of Chi-square contribution and deviation from independence (Figure 3).

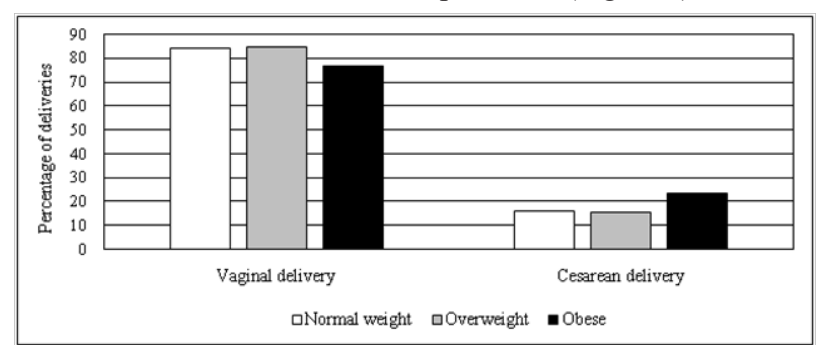

Figure 3 Route of delivery of primiparous patients by weight classification. Chi-square 8.104, Degrees of freedom 2, Probability level .02.

Gestational age at time of delivery and mean Apgar scores at one and five minutes were compared among the three weight classifications (Table 2). Normal weight adolescents were more likely to deliver preterm compared to overweight and obese teens $(30.0 \%$ versus $17.5 \%$ and $15.9 \%$, respectively, $\mathrm{P}<.001)$. Mean Apgar scores at five minutes, however, did not differ significantly between the groups.

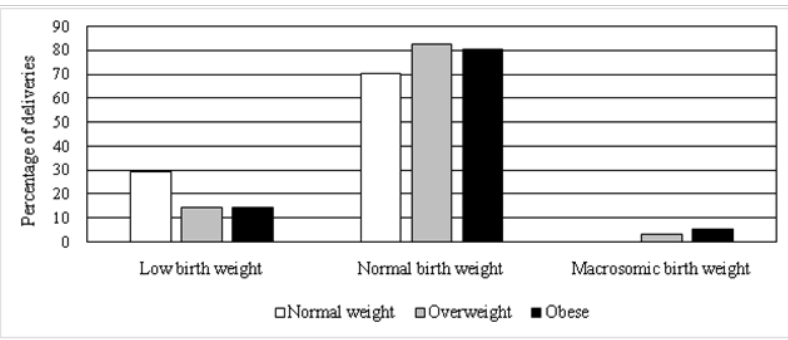

Figure 4 Neonatal birth weight by weight classification. Chi-square 36.846 , Degrees of freedom 4, Probability level .001.

Table I Demographic characteristics of study patients

\begin{tabular}{llll}
\hline & Normal weight & Overweight & Obese \\
\hline & $\mathrm{N}=220(2 \mathrm{I} .2 \%)$ & $\mathrm{N}=328(31.7 \%)$ & $\mathrm{N}=486(47.0 \%)$ \\
Mean age* & $18.1 \pm 1 . \mathrm{I}^{*}$ & $17.8 \pm 1.2$ & $17.8 \pm 1.2$ \\
Race Asian & $0(0 \%)$ & $2(0.4 \%)$ & $3(0.9 \%)$ \\
Black & $119(59.1 \%)$ & $194(60.7 \%)$ & $295(59.1 \%)$ \\
Hispanic & $17(7.7 \%)$ & $34(10.5 \%)$ & $51(10.4 \%)$ \\
White & $60(35.9 \%)$ & $95(27.8 \%)$ & $135(29.0 \%)$ \\
Undetermined & $5(2.3 \%)$ & $3(0.6 \%)$ & $2(0.8 \%)$ \\
Mean BMI $(\mathrm{kg} / \mathrm{m} 2)$ & $23.1 \pm 1.7$ & $27.7 \pm 1.5$ & $35.9 \pm 5.2$ \\
\hline
\end{tabular}

$$
* \mathrm{P}<.05
$$

Table 2 Neonatal outcomes by weight classification

\begin{tabular}{|c|c|c|c|}
\hline & Normal weight & Overweight & Obese \\
\hline & $\mathrm{N}=220(21.2 \%)$ & $\mathrm{N}=328(3 \mathrm{I} .7 \%)$ & $\mathrm{N}=486(47.0 \%)$ \\
\hline $\begin{array}{l}\text { Gestational age at } \\
\text { delivery (weeks) }\end{array}$ & $36.7 \pm 4.0 *$ & $38.1 \pm 2.6$ & $37.9 \pm 3.1$ \\
\hline $\begin{array}{l}\text { Mean I-minute Apgar } \\
\text { score }\end{array}$ & $7.4 \pm 1.9$ & $7.6 \pm 1.8$ & $7.3 \pm 2.2$ \\
\hline $\begin{array}{l}\text { Mean 5-minute Apgar } \\
\text { score }\end{array}$ & $8.4 \pm 1.4$ & $8.6 \pm 1.0$ & $8.4 \pm 1.2$ \\
\hline
\end{tabular}
$* \mathrm{P}<.001$

Neonatal birth weight was recorded as low birth weight $(<2500 \mathrm{~g})$, normal birth weight $(2500-3999 \mathrm{~g})$, and macrosomic $(>4000 \mathrm{~g})$. 
Concordant with gestational ages at time of delivery, normal weight adolescent mothers were more likely to have low birth weight infants than overweight and obese mothers (29.5\% versus $14.6 \%$ and $14.3 \%$, respectively, $\mathrm{P}<.001$ ) (Figure 4). Obese adolescent mothers, on the other hand, were more likely to give birth to macrosomic infants compared to normal and overweight mothers $(5.1 \%$ versus $0 \%$ and $3.0 \%$, respectively, $\mathrm{P}<.001$ ).

Prior studies have shown that adolescent mothers are less likely to require Cesarean section than adult mothers.4 Our study suggests that this effect of young maternal age may be lost in obese patients. Obese adolescents in our study were more likely to require a primary Cesarean delivery than their normal and overweight counterparts. Of all Cesarean deliveries performed in our study, obese patients appeared more likely to require a Cesarean delivery due to history of a prior Cesarean section or arrest of labor. Although not statistically significant in our study, this trend is concordant with studies of pregnancy outcomes in obese adults. Because our study only includes adolescent patients, we were unable to compare Cesarean delivery rates between adolescents and adults.

Studies of adolescent pregnancy have also shown that adolescent mothers are more likely to deliver low birth weight infants with lower Agar scores than their adult counterparts. ${ }^{2}$. Obesity similarly increases the risk of prematurity, but rather than low birth weight infants, obese mother are more likely to have macrosomic infants. ${ }^{7}$ Our study demonstrates that the weight of adolescents at time of delivery may significantly impact both of these outcomes. In contrast to what we know of adolescent pregnancy and pregnancy in the obese adult, obese adolescents in our study were actually less likely to deliver preterm. Similar to their obese adult counterparts, obese adolescents were less likely to deliver low birth weight infants and more likely to deliver macrosomic infants. Obesity confers an increased risk of gestational diabetes, which in turn increases the risk of macrosomia. We did not collect data on antenatal diagnoses, so we were unable to control for the role gestational diabetes may have played.

\section{Conclusion}

In conclusion, the weight of adolescents at time of delivery significantly impacts pregnancy outcomes. Obese adolescents in our study demonstrated some protection from risks of preterm delivery and low birth weight infants, however, they were at increased risk for macrosomia and Cesarean section.

\section{Acknowledgments}

None.

\section{Conflicts of interest}

The authors declare no conflicts of interest.

\section{References}

1. National snapshot. The National Campaign to Prevent Teen and Unplanned Pregnancy. 2015.

2. Fraser A, Brockert J, Ward R. Association of young maternal age with adverse reproductive outcomes. New England $J$ of Med. 1995;332(17):1113-1117.

3. Phipps M, Sowers M. Defining early adolescent childbearing. Am J Pub Health. 2002;92(1):125-128.

4. Lubarsky S, Schiff E, Friedman S, et al. Obstetric characteristics among nulliparas under age 15. Obst \& Gyn. 1994;84(3):365-368.

5. Koniak-Griffin D, Turner-Pluta C. Health risks and psychosocial outcomes of early childbearing:a review of the literature. J Perinat Neonatal Nurs. 2001;15(2):1-17.

6. Ogden CL, Carroll MD, Kit BK, et al. Prevalence of childhood and adult obesity in the United States, 2011-2012. JAMA. 2014;311(8):806-814.

7. CDC Growth Charts. National Center for Health Statistics at the Center for Disease Control and Prevention. 2000.

8. American College of Obstetricians and Gynecologists. Committee Opinion No. 549:Obesity in Pregnancy. Obstet Gynecol. 2013;121:213217 